\title{
Does adding local salvage ablation therapy provide survival advantage for patients with locally recurrent prostate cancer following radiotherapy? Whole gland salvage ablation post-radiation failure in prostate cancer
}

\author{
Shiva Madhwan Nair ${ }^{1}$; Andrew Warner ${ }^{2}$; Arnon Lavi ${ }^{1}$; George Rodrigues ${ }^{2}$; Joseph Chin ${ }^{1}$ \\ ${ }^{1}$ Departments of Urology and Oncology, Western University, London, ON, Canada; ${ }^{2}$ Department of Radiation \\ Oncology, Western University, London, ON, Canada
}

Cite as: Nair SM, Warner A, Lavi A, et al. Does adding local salvage ablation therapy provide survival advantage for patients with locally recurrent prostate cancer following radiotherapy? Can Urol Assoc J 2020 September 28; Epub ahead of print.

http://dx.doi.org/10.5489/cuaj.6676

Published online September 28, 2020

$* * *$

Abstract

Introduction: Some men who experience prostate cancer recurrence post-radiotherapy may be candidates for local salvage therapy, avoiding and delaying systemic treatments. Our aim was to assess the impact of clinical outcomes of adding salvage local treatment in prostate cancer patients who have failed radiation therapy.

Methods: Following radiation biochemical failure, salvage transperineal cryotherapy (sCT, $\mathrm{n}=186$ ), transrectal high intensity focused ultrasound ablation ( $\mathrm{sHIFU}, \mathrm{n}=113$ ) or no salvage treatment (NST, identified from the pan-Canadian Prostate Cancer Risk Stratification [ProCaRS] database $[\mathrm{n}=982]$ ) were compared with propensity-score matching. Primary endpoints were cancer-specific survival (CSS) and overall survival (OS).

Results: Median followup was 11.6, 25.1, and 14.3 years following NST, sCT, and SHIFU, respectively. Two propensity-score matched analyses were performed: 1) 196 NST vs. 98 sCT; and 2) 177 NST vs. 59 sHIFU. In the first comparison, there were 78 deaths and 49 prostate cancer deaths for NST vs. 80 deaths and 24 prostate cancer deaths for sCT. There were significant benefits in CSS $(\mathrm{p}<0.001)$ and OS $(\mathrm{p}<0.001)$ favoring $\mathrm{sCT}$. In the second comparison, there were 52 deaths (31 from prostate cancer) for NST vs. 18 deaths (nine from prostate cancer) for sHIFU. There were no significant differences in CSS or OS possibility attributed to reduced sample size and shorter followup of sHIFU cohort.

Conclusions: In select men with recurrent prostate cancer post-radiation, further local treatment may lead to benefits in CSS. These hypothesis-generating findings should ideally be validated in a prospective clinical trial setting. 


\section{Introduction}

Localized prostate cancer can be treated with curable intent with radiation therapy. However, a third of the patients fail with biochemical recurrence. ${ }^{1,2}$ These patients are usually treated with systemic androgen deprivation therapy (ADT), with its associated comorbidity. ${ }^{3,4}$ Some of the patients with localized recurrence post radiation may be able to avoid or delay systemic therapy with salvage local treatment options. Local ablation options include salvage cryotherapy ( $\mathrm{sCT}$ ) and salvage high intensity focused ultrasound (sHIFU). These ablation techniques may be offered to patients who are averse to the morbidity of salvage radical surgery or are not suitable surgical candidates. $\mathrm{SCT}$ has low overall complications and a biochemical cure rate of $39 \%$ at 10 years. ${ }^{5,6}$ sHIFU and sCT have been shown to have a similar comorbidity profile. ${ }^{7}$

Whilst there are reports on comparing oncological outcomes with ablation technique versus salvage prostatectomy, ${ }^{8}$ there are no reports with local salvage treatment versus those without salvage therapy (NST). Herein, we performed a propensity-score matched analysis with a large multi-institutional radiation therapy database (where patients received standard of care but did not have salvage local prostate treatment) compared with a prospective singleinstitution database on salvage local ablative therapies, to examine for differences in cancer specific survival (CSS) and overall survival (OS). Ideally, the findings of this analysis would provide both guidance for clinicians and clinical trialists in the management of this challenging patient population.

\section{Methods}

\section{Patient populations}

The ablation therapy patient cohorts were selected from a single academic centre, with a prospectively maintained database. Patients underwent sCT between 1994 and 2004 (n=186) or sHIFU between 2006 and $2018(\mathrm{n}=113)$ performed by a single surgeon (JC), as previously described. ${ }^{9,10}$ All patients had previously received primary radiotherapy (external beam radiation therapy (EBRT) or brachytherapy) and had histologically proven radio-recurrent prostate cancer (all patients had a trans-rectal ultrasound guided prostate biopsy postradiation confirming recurrence). All patients except two had metastatic screening with radionucleotide bone scan and abdominal and pelvic computed tomography (CT). Some patients had been started on ADT prior to their referral for local ablative therapy and were included in the salvage cohorts. However, ADT was discontinued immediately following both $\mathrm{SCT}$ and SHIFU.

The NST patient cohort was selected from the Prostate Cancer Risk Stratification (ProCaRS) database consisting of patients from four Canadian institutions including 3440 prostate cancer patients treated with EBRT between 1994 and 2010, as discussed in detail previously. ${ }^{11,12}$ This population was further restricted to patients subsequently developing biochemical failure accordingly to the American Society for Radiation Oncology - Radiation Therapy Oncology Group Phoenix II definition involving a prostate specific antigen (PSA) 
rise of $\geq 2 \mathrm{ng} / \mathrm{mL}$ above the nadir PSA ( $\mathrm{n}=982) .{ }^{13}$ Research Ethics Board approval was obtained from Western University for this study (REB \#103538).

\section{Treatment}

SCT was performed, as previous described, ${ }^{10}$ with transperineal placement of cryotherapy probes under 3-dimensional transrectal ultrasound guidance. Two freeze-thaw cycles were employed. sHIFU treatment was performed with the Sonablate ${ }^{\circledR} 500$ device with continuous trans-rectal monitoring. ${ }^{9}$ A suprapubic Foley catheter, placed intraoperatively, was used to divert urine for 3 weeks for both groups.

\section{Followup}

For the ablation group, follow-up data was obtained from clinical records or by contacting patients directly. Clinical monitoring outside 24 months was at the discretion of the referring urologists. ${ }^{14}$ In case of biochemical or clinical failure following local ablative therapy, initiation of ADT was at the discretion of the treating physician.

\section{Endpoints}

The primary end-point was CSS calculated as time from date of radiotherapy to date of death attributed to prostate cancer, or date of last follow-up, whichever comes first. The secondary end-point was OS calculated as time from date of radiotherapy to date of death (any cause), or date of last follow-up, whichever comes first. As a sensitivity analysis, both end-points were calculated as time from date of biochemical failure (for NST cohort) or from date of salvage treatment (for $\mathrm{SCT}$ and sHIFU cohorts).

\section{Statistical analysis}

Patients receiving NST were matched to sCT (match \#1) and separately to sHIFU (match \#2) using propensity-score matching. Propensity scores were generated using multivariable logistic regression (propensity score model) predictive of treatment assignment (NST vs. sCT for match \#1 and NST vs. sHIFU for match \#2) based on: age at radiation; pre-radiation baseline PSA; pre-radiation Gleason score $(<6,6,7$ or $8-10)$; pre-radiation T-stage (cT1, cT2, cT3, cT4); and pre-radiation ADT (yes, no). The Gleason Score of $<6$ was used as documented by the original pathology reports (which had not conformed to current pathology classifications). Patients were risk-stratified according to the Genitourinary Radiation Oncologists of Canada (GUROC) ${ }^{12}$ :

1) Low risk: T1c-T2a, PSA $\leq 10 \mathrm{ng} / \mathrm{mL}$ and Gleason score $\leq 6$

2) Intermediate risk: T1-T2, PSA $\leq 20 \mathrm{ng} / \mathrm{mL}$, and Gleason score $\leq 7$, not otherwise low risk

3) High risk: T3-T4 or PSA > 20ng/mL, or Gleason score 8-10

All possible interaction terms were examined and retained in final models if significant. Pre-radiation ADT was defined as starting prior to completion of radiation for all cohorts. Twelve possible matching scenarios were examined for each match based on pre-specified ratios (1:1 to $1: 4)$ and calipers $(0.2$ of standard deviation of the logit of the propensity score; 0.10 and 0.15$).{ }^{15}$ Standardized differences were calculated to assess for imbalance in baseline 
characteristics included in the propensity score model prior to matching and for each match scenario selecting a threshold of $>0.10$ to indicate statistical imbalance. ${ }^{16,17}$ Final optimal matches were selected based minimizing overall standardized differences across all variables included in match and maximizing power (sample size). Match \#1 (NST vs. sCT) was based on a caliper of 0.2 of standard deviation of the logit of the propensity score and Match \#2 (NST vs. SHIFU) was based on a caliper of 0.15 .

Descriptive statistics were generated for baseline patient characteristics stratified by cohort for all patients prior to matching for Match \#1 (NST ( $\mathrm{n}=982)$ vs. sCT $(\mathrm{n}=186)$ and Match \#2 (NST ( $\mathrm{n}=982)$ vs. sHIFU $(\mathrm{n}=113)$ ) and repeated for matched cohorts. Comparisons were made using the Chi-square test, Fisher's Exact test, two-sample T-test or Wilcoxon rank sum test as appropriate. For variables included in the propensity-score model, comparisons were made using the paired T-test, Wilcoxon signed rank test or McNemar test as appropriate in addition to calculation of standardized differences (discussed previously). Kaplan-Meier estimates were generated for CSS and OS stratified by matched cohorts (Match \#1: NST vs. sCT; Match \#2: NST vs. sHIFU) and compared using the stratified log-rank test (stratified by matched pair number). All statistical analysis was performed using SAS version 9.4 software (SAS Institute Inc., Cary, NC, USA), using two-sided statistical testing at the 0.05 significance level.

\section{Results}

\section{Salvage cryotherapy post radiation vs. no salvage treatment}

Baseline characteristics comparing sCT and NST are summarized in Table 1. Prior to matching, compared to NST, patients receiving sCT were younger $(\mathrm{p}<0.001)$, had more cT2 and fewer cT3 and cT4 disease $(\mathrm{p}<0.001)$, and with more Gleason score 6 and fewer Gleason score 7-10 disease $(\mathrm{p}<0.001)$. Fewer $\mathrm{sCT}$ patients received pre-radiation ADT $(\mathrm{p}<$ $0.001)$. The median follow-up from initial radiation was 11.6 years $(95 \%$ confidence interval [CI]: 11.3-11.9) for NST and 25.1 years (95\% CI: 24.2-26.1) for the sCT group. Propensityscore matching resulted in final matched cohorts of $98 \mathrm{sCT}$ and 196 NST patients in a 1:2 ratio. Details of final matched cohort selection are summarized in Supplemental Table 1. Following propensity-score matching, cohorts were comparable in terms of variables included in propensity score model (age at radiation, pre-radiation baseline PSA, preradiation T-stage, pre-radiation Gleason score and ADT pre-radiation) with no significant differences observed.

In the NST cohort there were 78 deaths with 49 from prostate cancer (median followup 11.6 years) compared to 80 deaths with 24 from prostate cancer in the sCT cohort (median follow-up 25.1 years). This translated to a significantly improved CSS $(\mathrm{p}<0.001)$ and OS ( $\mathrm{p}$ $<0.001$ ) in patients receiving sCT compared to NST without local salvage therapy (fig. 1) calculated from date of radiotherapy. Median OS was 12.3 years for NST and 16.3 years for SCT (median CSS not reached). This effect was still present following a sensitivity analysis calculated from date of biochemical failure post radiation (for NST cohort) and from date of salvage ablation treatment (for $\mathrm{sCT}$ cohort) for CSS $(\mathrm{p}=0.003)$ and OS $(\mathrm{p}=0.004)$ 
(Supplement fig. 1). Following sensitivity analysis, median CSS was 11.6 years for NST (not reached for $\mathrm{SCT}$ ), median OS was 8.4 years for NST and 11.8 years for $\mathrm{sCT}$.

\section{Salvage high intensity focused ultrasound (HIFU) ablation post radiation versus no salvage treatment}

Baseline characteristics comparing sHIFU and NST are summarized in Table 2. Similarly, prior to matching compared to NST, patients receiving SHIFU were younger $(p<0.001)$, had more T1 and fewer T3 disease $(\mathrm{p}<0.001)$, with more Gleason score 6 and fewer Gleason score $8-10$ disease $(\mathrm{p}<0.001)$. Fewer sHIFU patients received pre- and post-radiation ADT ( $\mathrm{p}<0.001$ and $\mathrm{p}<0.001$ respectively). Median follow-up from initial radiation was 14.3 years (95\% CI: 13.3-16.3) for sHIFU patients. Propensity-score matching resulted in final matched cohorts of 59 sHIFU and 177 NST patients in a 1:3 ratio. Details of final matched cohort selection are summarized in Supplemental Table 1. Following propensity-score matching, cohorts were comparable in terms of variables included in propensity score model (age at radiation, pre-radiation baseline PSA, pre-radiation T-stage, pre-radiation Gleason score and ADT pre-radiation) with no significant differences observed.

Fifty-two deaths with 31 from prostate cancer were observed in the NST cohort (median follow-up 11.6 years) compared with 18 deaths with 9 from prostate cancer in the sHIFU cohort (median follow-up 14.3 years). However, there were no significant differences in CSS ( $p=0.326)$ or OS ( $p=0.182$ ) calculated from date of radiotherapy (fig. 2). Median OS was 12.8 years for NST and 17.4 years for sHIFU (median CSS not reached). Calculating OS and CSS from date of biochemical failure (for NST cohort) and from date of salvage ablation treatment (for sHIFU cohort) did not result in any significant difference in CSS ( $\mathrm{p}=$ 0.639 ) or OS ( $p=0.937$ ) (Supplementary fig. 2). Median OS was 9.8 years for NST and 10.4 years for sHIFU (median CSS not reached).

\section{Discussion}

This study showed that long-term outcomes favour salvage treatment following radiation therapy failure for prostate cancer when compared to NST with no further local treatment. CSS and OS were significantly lower in the NST cohort when compared to the sCT cohort. The low number of events in the smaller sHIFU cohort highlights the need for longer followup for differences in OS and CSS (sHIFU median follow-up 14.3 years vs. 25.1 years for $\mathrm{SCT}$ ). The sHIFU cohort findings are thus relatively exploratory in comparison to the very mature sCT cohort. This study is the first to compare long-term outcomes of salvage local treatment to NST without further local therapy.

The patients who had biochemical failure in the NST group would be treated by systemic (ADT) at the discretion of their physician. The use of ADT within this cohort of patients is not known, but previous studies have shown $94 \%$ of radiation failure patients were treated with only ADT. ${ }^{4}$ Some of these patients would have potentially avoided ADT use entirely if the localized recurrence was successfully eradicated. Within the sCT cohort we have previously shown $58 \%$ had delayed biochemical failure and $51 \%$ requiring ADT. ${ }^{18}$ Systemic ADT has been linked with increased cardiovascular, metabolic and bone-related 
morbidity, which is an important consideration given these patients are already at increased risk on account of their demographics and underlying malignancy. ${ }^{3,19}$

On the other hand, patients undergoing salvage therapies post-radiation may be subjected to significant local adverse effects. Serious complications from $\mathrm{sCT}$ include rectourethral fistulas (3\%) and severe incontinence $(7 \%) .{ }^{20}$ Most common complications are usually manageable endoscopically. ${ }^{21}$ Erectile dysfunction is also common with increasing age, comorbidities and the original local radiation as likely contributing factors. ${ }^{6}$ Previously, we have found that SHIFU has an improved morbidity profile when compared to sCT, as the ablation energy can be more precisely targeted to the prostate tissue. ${ }^{10}$ Thus, even though the adverse effects can be significant, the incidence is relatively low and most can be managed without significant additional morbidity.

Patients in the ablation group had repeat biopsy within 12 months. Our series had $15 \%$ local persistent disease, ${ }^{22}$ and in the absence of metastatic disease were offered repeat ablation. Thus, the majority of treatment failure in the ablation group were systemic in nature. Since the salvage ablation cohorts were screened only with computerized axial tomography and radionucleotide bone scans prior to ablation, the systemic failure rate should improve with better staging modalities such as positron emission tomography (PET), especially when contemporary radionuclide tracers such as Prostate Specific Membrane Antigen (PSMA) are used. Hopefully this can result in further reduction in the detection of occult metastatic disease, ${ }^{23}$ thereby refining the patient selection process for local salvage ablative procedures, whilst improving the overall success rate of local salvage therapies.

Selection of an ideal patient who would benefit most from salvage therapies is key to avoid side-effects of local ablation whilst also preventing systemic progression. Previous studies have shown pre-salvage Gleason score and PSA were useful in predicting treatment response to the salvage therapy. Similarly, once salvage cryotherapy treatment was administered, a lower PSA nadir predicted decreased biochemical recurrence. ${ }^{5,24}$

There are several limitations to the study. Limitations specific to the NST cohort described previously include heterogeneity within the cohort with regards to treatment regimen. ${ }^{11}$ The heterogeneity within the cohort has been highlighted as a concern especially with one site potentially over-classifying CSS. Likewise, the limitations of salvation ablation group include the retrospective and single-surgeon nature of this study. ${ }^{10}$ Furthermore, data on salvage ADT with the NST group were not available. There is a very low chance of the NST group undergoing further local therapy that was not captured. However, salvage ablation was not readily available to our NST population. Comorbidity data was not available for the NST and not included in the propensity-score model. This would impact the comparisons between the cohorts for the OS. The cohort sizes, follow-up duration and treatment time were different. The sCT and sHIFU were done in sequential eras, hence the groups were not merged into a larger single group. Furthermore, the primary analyses compared patients from date of radiation. Within the ablation group there could be a selection bias, as some of the patients eligible for ablation may have died prior to selection. This was attempted to be corrected with comparing the groups from date of biochemical failure in the NST group and date of ablation therapy (date of biochemical failure for salvage group was not available for 
all patients). The analyses results were similar, thus indicating the selection bias as likely having minimal impact. During the study period from 1994 to present, there has been significant amount of changes in prostate cancer management, including some of the propensity score matching variables such as Gleason score, thus making the results hypothesis generating. To overcome the limitations of the study (vide supra), a prospective external validation is vital. However, recruiting such patients in a timely manner may not be feasible, with both the paucity of appropriate patients and long follow up duration required for oncological end points to reach. Furthermore, a prospective study of such duration would be similarly affected by future modifications in prostate cancer management in the nonsalvage treatment cohort. Therefore, the authors chose to take advantage of the long followup from our prospectively accrued local salvage cohort and attempted to address some of the deficiencies with propensity-score matching, albeit with the limitations discussed.

\section{Conclusions}

In selected men with salvage therapy to the prostate bed following recurrence of prostate cancer may offer improvement in important clinical outcomes compared to current standard of care. These findings would be ideally validated in a prospective randomized control trial.

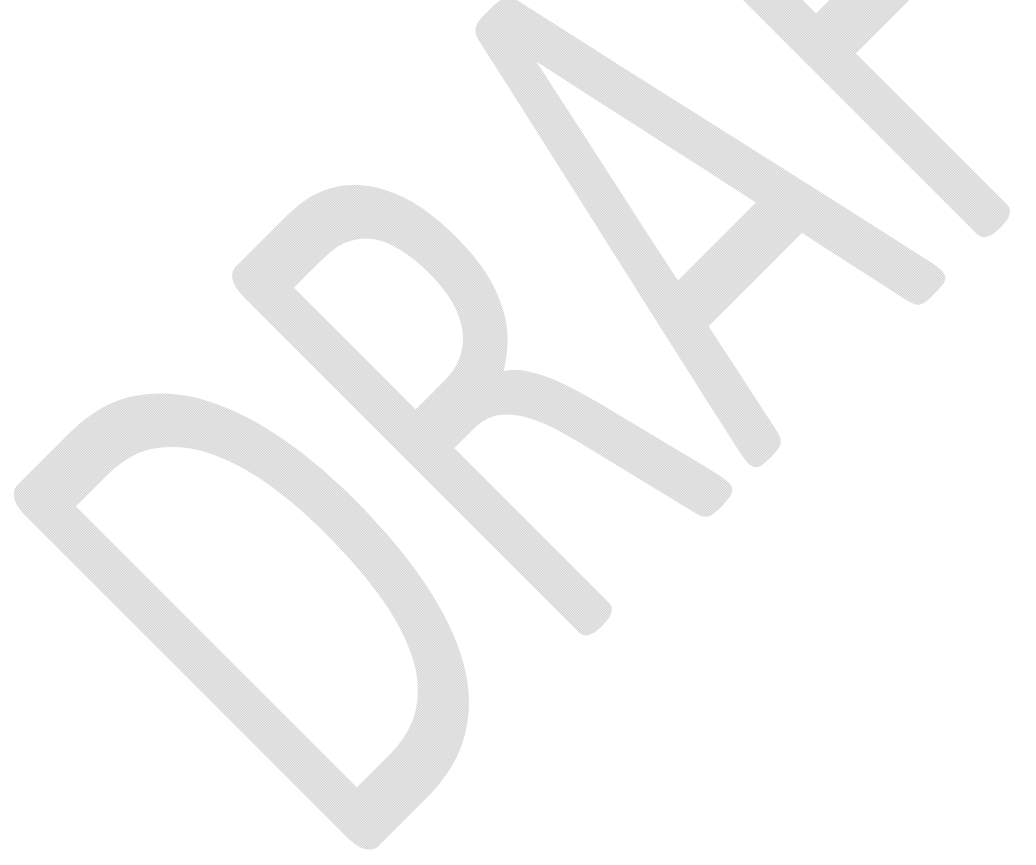




\section{References}

1. Zietman AL, Bae K, Slater JD et al. Randomized trial comparing conventional-dose with high-dose conformal radiation therapy in early-stage adenocarcinoma of the prostate: long-term results from proton radiation oncology group/american college of radiology 95-09. J Clin Oncol 2010; 28: 1106-1111. 10.1200/JCO.2009.25.8475

2. Zelefsky MJ, Eastham JA, Cronin AM et al. Metastasis after radical prostatectomy or external beam radiotherapy for patients with clinically localized prostate cancer: a comparison of clinical cohorts adjusted for case mix. J Clin Oncol 2010; 28: 15081513. 10.1200/JCO.2009.22.2265

3. Beckmann K, Garmo H, Adolfsson J et al. Androgen Deprivation Therapies and Changes in Comorbidity: A Comparison of Gonadotropin-releasing Hormone Agonists and Antiandrogen Monotherapy as Primary Therapy in Men with High-risk Prostate Cancer. Eur Urol 2018. 10.1016/j.eururo.2018.11.022

4. Agarwal PK, Sadetsky N, Konety BR, Resnick MI, Carroll PR, Cancer of the Prostate Strategic Urological Research E. Treatment failure after primary and salvage therapy for prostate cancer: likelihood, patterns of care, and outcomes. Cancer 2008; 112: 307-314. 10.1002/cncr.23161

5. Williams AK, Martinez CH, Lu C, Ng CK, Pautler SE, Chin JL. Disease-free survival following salvage cryotherapy for biopsy-proven radio-recurrent prostate cancer. Eur Urol 2011; 60: 405-410. 10.1016/j.eururo.2010.12.012

6. Golbari NM, Katz AE. Salvage Therapy Options for Local Prostate Cancer Recurrence After Primary Radiotherapy: a Literature Review. Curr Urol Rep 2017; 18: 63. 10.1007/s11934-017-0709-4

7. Autran-Gomez AM, Scarpa RM, Chin J. High-intensity focused ultrasound and cryotherapy as salvage treatment in local radio-recurrent prostate cancer. Urol Int 2012; 89: 373-379. 10.1159/000339616

8. Pisters LL, Leibovici D, Blute $\mathrm{M}$ et al. Locally recurrent prostate cancer after initial radiation therapy: a comparison of salvage radical prostatectomy versus cryotherapy. J Urol 2009; 182: 517-525; discussion 525-517. 10.1016/j.juro.2009.04.006

9. Siddiqui KM, Billia M, Arifin A, Li F, Violette P, Chin JL. Pathological, Oncologic and Functional Outcomes of a Prospective Registry of Salvage High Intensity Focused Ultrasound Ablation for Radiorecurrent Prostate Cancer. J Urol 2017; 197 : 97-102. 10.1016/j.juro.2016.06.092

10. Siddiqui KM, Billia M, Williams A, Alzahrani A, Chin JL. Comparative morbidity of ablative energy-based salvage treatments for radio-recurrent prostate cancer. Can Urol Assoc J 2015; 9: 325-329. 10.5489/cuaj.3113

11. Rodrigues G, Lukka H, Warde P et al. The prostate cancer risk stratification (ProCaRS) project: recursive partitioning risk stratification analysis. Radiother Oncol 2013; 109: 204-210. 10.1016/j.radonc.2013.07.020

12. Rodrigues G, Lukka H, Warde P et al. The prostate cancer risk stratification project: database construction and risk stratification outcome analysis. J Natl Compr Canc Netw 2014; 12: 60-69.

13. Roach M, 3rd, Hanks G, Thames H, Jr. et al. Defining biochemical failure following radiotherapy with or without hormonal therapy in men with clinically localized prostate cancer: recommendations of the RTOG-ASTRO Phoenix Consensus Conference. Int J Radiat Oncol Biol Phys 2006; 65: 965-974.

10.1016/j.ijrobp.2006.04.029 
14. Amling CL, Bergstralh EJ, Blute ML, Slezak JM, Zincke H. Defining prostate specific antigen progression after radical prostatectomy: what is the most appropriate cut point? J Urol 2001; 165: 1146-1151.

15. Austin PC. Some methods of propensity-score matching had superior performance to others: results of an empirical investigation and Monte Carlo simulations. Biom J 2009; 51: 171-184. 10.1002/bimj.200810488

16. Austin PC, Grootendorst P, Anderson GM. A comparison of the ability of different propensity score models to balance measured variables between treated and untreated subjects: a Monte Carlo study. Stat Med 2007; 26: 734-753. 10.1002/sim.2580

17. Imai K, King G, Stuart EA. Misunderstandings between experimentalists and observationalists about causal inference. J R Statist Soc A 2008; 171: 481-502.

18. Nair SM, Peters M, Abed H, van der Voort van Zyp J, van Son M, Chin J. Tumor control outcomes of salvage cryotherapy for radiorecurrent prostate cancer at median 12 years follow-up. J Urol 2019; 201: e1142.

https://doi.org/10.1097/01.JU.0000557335.01833.fc

19. Saylor PJ, Smith MR. Metabolic complications of androgen deprivation therapy for prostate cancer. J Urol 2009; 181: 1998-2006; discussion 2007-1998. 10.1016/j.juro.2009.01.047

20. Chin JL, Pautler SE, Mouraviev V, Touma N, Moore K, Downey DB. Results of salvage cryoablation of the prostate after radiation: identifying predictors of treatment failure and complications. J Urol 2001; 165: 1937-1941; discussion 1941-1932.

21. Vora A, Agarwal V, Singh P et al. Single-institution comparative study on the outcomes of salvage cryotherapy versus salvage robotic prostatectomy for radioresistant prostate cancer. Prostate Int 2016; 4: 7-10. 10.1016/j.prnil.2015.11.002

22. Chin JL, Touma N, Pautler SE et al. Serial histopathology results of salvage cryoablation for prostate cancer after radiation failure. J Urol 2003; 170: 1199-1202. 10.1097/01.ju.0000085620.28141.40

23. Ceci F, Castellucci P, Graziani T et al. (68)Ga-PSMA-11 PET/CT in recurrent prostate cancer: efficacy in different clinical stages of PSA failure after radical therapy. Eur J Nucl Med Mol Imaging 2019; 46: 31-39. 10.1007/s00259-018-4189-7

24. Spiess PE, Levy DA, Mouraviev V, Pisters LL, Jones JS. Predictors of biochemical failure in patients undergoing prostate whole-gland salvage cryotherapy: a novel risk stratification model. BJU Int 2013; 112: E256-261. 10.1111/j.1464-

410X.2012.11695.X 


\section{Figures and Tables}

Fig. 1. Kaplan-Meier plot for survival outcomes for matched patients (NST and $\mathrm{sCT}$ ) from date of radiation therapy. NST: no salvage treatment; $\mathrm{SCT}$ : salvage transperineal cryotherapy.
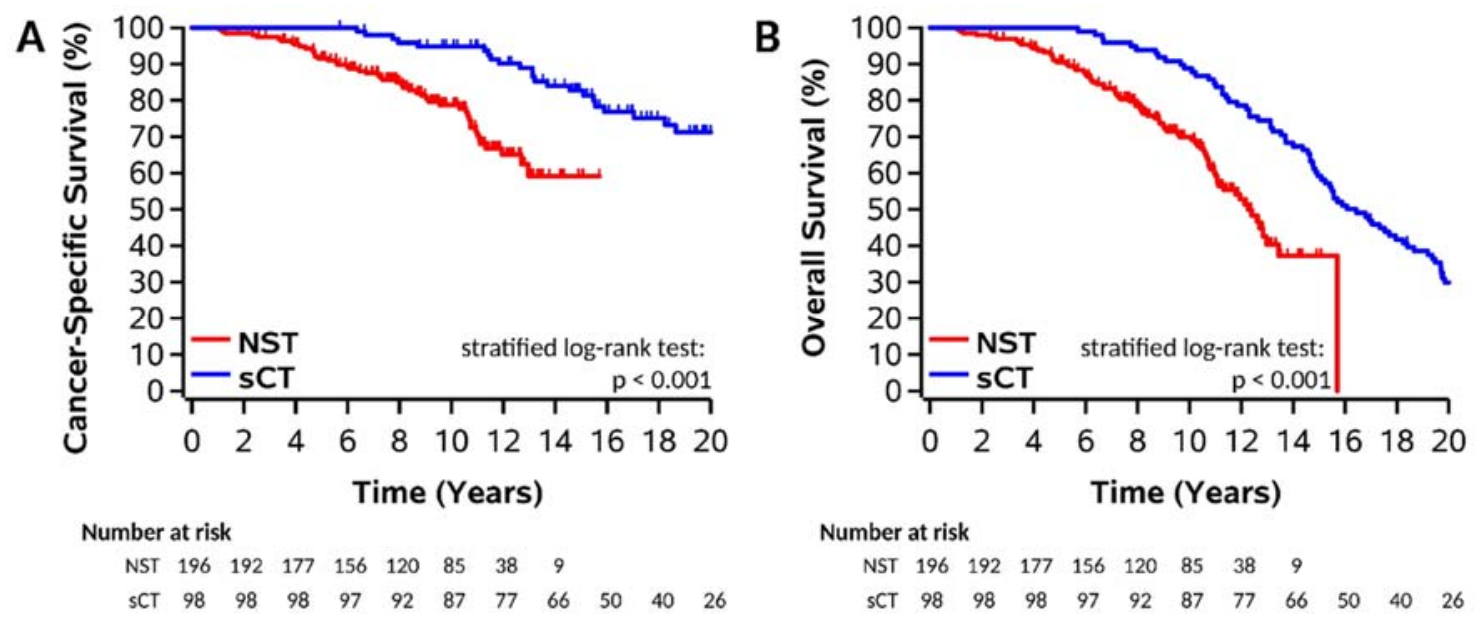

Fig. 2. Kaplan-Meier plot for survival outcomes for matched patients (NST and SHIFU) from date of radiation therapy. NST: no salvage treatment; sHIFU: salvage transrectal high intensity focused ultrasound ablation.
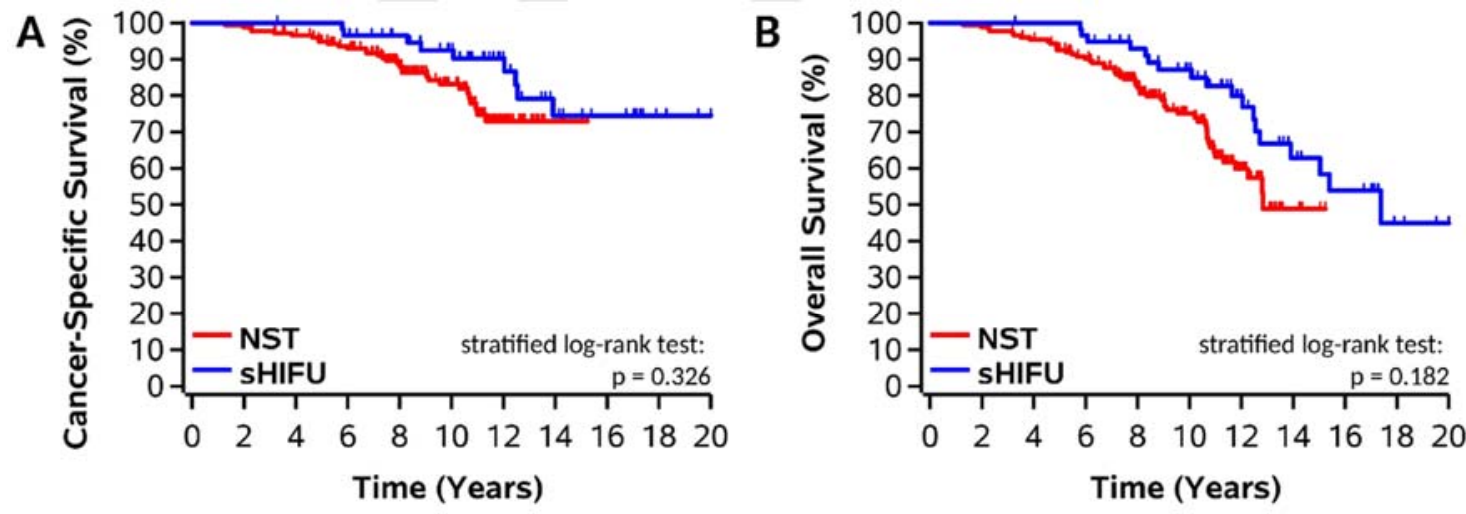

Number at risk

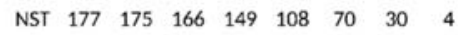

Number at risk
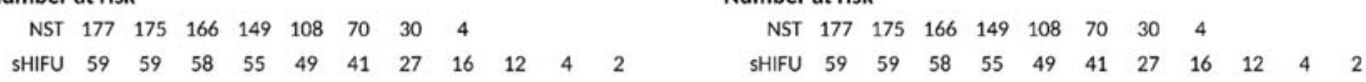


\begin{tabular}{|c|c|c|c|c|c|c|c|c|c|c|}
\hline \multirow[b]{2}{*}{ Characteristic } & \multicolumn{5}{|c|}{ All patients $(n=1168)$} & \multicolumn{5}{|c|}{ Matched patients $(n=294)$} \\
\hline & $\mathbf{n}$ & $\begin{array}{c}\text { NST } \\
(\mathrm{n}=982)\end{array}$ & $\begin{array}{c}\mathrm{sCT} \\
(\mathrm{n}=186)\end{array}$ & p & SD & $\mathbf{n}$ & $\begin{array}{c}\text { NST } \\
(n=196)\end{array}$ & $\begin{array}{c}\mathrm{sCT} \\
(\mathrm{n}=98)\end{array}$ & $\mathbf{p}$ & SD \\
\hline $\begin{array}{l}\text { Age at radiation, }{ }^{1} \text { mean } \pm \\
\mathrm{SD}, \text { median }(\mathrm{IQR})\end{array}$ & 1168 & $\begin{array}{c}70.36 .5 \\
71.0(67.0 \\
75.0) \\
\end{array}$ & $\begin{array}{c}64.6 \pm 6.1 \\
65.4(60.3 \\
69.4) \\
\end{array}$ & $<0.001$ & 0.892 & 294 & $\begin{array}{c}67.8 \pm 6.5 \\
69.0(63.0 \\
73.0) \\
\end{array}$ & $\begin{array}{c}67.9 \pm 4.4 \\
68.4(65.4 \\
70.9) \\
\end{array}$ & 0.788 & 0.027 \\
\hline $\begin{array}{l}\text { Baseline PSA (pre- } \\
\text { radiation) },{ }^{1} \text { mean } \pm \mathrm{SD}, \\
\text { median (IQR) }\end{array}$ & 1133 & $\begin{array}{c}18.7 \pm 22.8 \\
11.9(7.3 \\
20.0) \\
\end{array}$ & $\begin{array}{c}15.8 \pm 14.7 \\
11.0(7.7 \\
18.9) \\
\end{array}$ & 0.221 & 0.150 & 294 & $\begin{array}{c}16.9 \pm 20.3 \\
12.7(7.0 \\
18.8) \\
\end{array}$ & $\begin{array}{c}15.0 \pm 12.6 \\
11.0(7.7 \\
17.0) \\
\end{array}$ & 0.459 & 0.108 \\
\hline $\begin{array}{l}\text { T stage (pre-radiation) }{ }^{1}, \mathrm{n} \\
(\%)\end{array}$ & 1131 & & & $<0.001$ & & 294 & & & 0.304 & \\
\hline $\mathrm{T} 1$ & & $166(17.2)$ & $33(19.8)$ & 0.426 & 0.065 & & $37(18.9)$ & $20(20.4)$ & 0.701 & 0.039 \\
\hline $\mathrm{T} 2$ & & $482(50.0)$ & $113(67.7)$ & $<0.001$ & 0.365 & & $126(64.3)$ & $61(62.2)$ & 0.642 & 0.042 \\
\hline T3 & & $288(29.9)$ & $21(12.6)$ & $<0.001$ & 0.433 & & $33(16.8)$ & $17(17.4)$ & 0.879 & 0.014 \\
\hline $\mathrm{T} 4$ & & $28(2.9)$ & $0(0)$ & 0.026 & 0.245 & & $0(0)$ & $0(0)$ & - & - \\
\hline $\begin{array}{l}\text { Gleason score (pre- } \\
\text { radiation })^{1}, \mathrm{n}(\%)\end{array}$ & 1125 & & & $<0.001$ & & 294 & & & 0.406 & \\
\hline$<6$ & 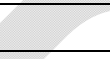 & $180(18.8)$ & $2(1.2)$ & $<0.001$ & 0.615 & & $3(1.5)$ & $2(2.0)$ & 0.706 & 0.039 \\
\hline 6 & & $212(22.2)$ & $101(59.8)$ & $<0.001$ & 0.827 & & $100(51.0)$ & $51(52.0)$ & 0.796 & 0.020 \\
\hline 7 & & $398(41.6)$ & $54(32.0)$ & 0.018 & 0.202 & & $66(33.7)$ & $35(35.7)$ & 0.659 & 0.043 \\
\hline $8-10$ & tre & $166(17.4)$ & $12(7.1)$ & $<0.001$ & 0.317 & & $27(13.8)$ & $10(10.2)$ & 0.250 & 0.110 \\
\hline GUROC risk, n (\%) & 1137 & & & 0.003 & - & 294 & & & 0.970 & - \\
\hline Low & & $122(12.4)$ & $34(21.9)$ & & & & $33(16.8)$ & $17(17.4)$ & & \\
\hline Intermediate & & $398(40.5)$ & $63(40.7)$ & & & & $89(45.4)$ & $43(43.9)$ & & \\
\hline High & & $462(47.1)$ & $58(37.4)$ & & & & $74(37.8)$ & $38(38.8)$ & & \\
\hline ADT pre-radiation, ${ }^{1} \mathrm{n}(\%)$ & 1168 & $397(40.4)$ & $33(17.7)$ & $<0.001$ & 0.516 & 294 & $69(35.2)$ & $27(27.6)$ & 0.092 & 0.165 \\
\hline
\end{tabular}


Nair et al

Role of salvage ablation post-radiation failure

\begin{tabular}{|l|c|c|c|c|c|c|c|c|c|}
\hline Unknown & & $29(6.2)$ & $9(6.2)$ & & & & $3(3.9)$ & $4(5.0)$ & \\
\hline $\begin{array}{l}\text { Median followup (years), } \\
\text { median (95\% CI) }\end{array}$ & 1168 & $\begin{array}{c}11.6(11.3, \\
11.9)\end{array}$ & $\begin{array}{c}25.1(24.2, \\
26.1)\end{array}$ & $<\mathbf{0 . 0 0 1}$ & - & 294 & $\begin{array}{c}11.2(10.5, \\
11.8)\end{array}$ & $\begin{array}{c}22.7(21.4, \\
24.6)\end{array}$ & $<\mathbf{0 . 0 0 1}$ \\
\hline
\end{tabular}

${ }^{1}$ Included in propensity-score model; ${ }^{2}$ calculated using reverse Kaplan-Meier method; $p<0.05$ and SD $>0.10$ shown in BOLD. ADT: androgen deprivation therapy; CI: confidence interval; GUROC: Genitourinary Radiation Oncologists of Canada; IQR: interquartile range; NST: no salvage treatment; PSA: prostate-specific antigen; sCT: salvage transperineal cryotherapy; SD: standardized deviation. 
Table 2. Baseline patient characteristics stratified by cohort (NST vs. sHIFU)

\begin{tabular}{|c|c|c|c|c|c|c|c|c|c|c|}
\hline \multirow[b]{2}{*}{ Characteristic } & \multicolumn{5}{|c|}{ All patients $(n=1095)$} & \multicolumn{5}{|c|}{ Matched patients $(n=236)$} \\
\hline & $\mathbf{n}$ & $\begin{array}{c}\text { NST } \\
(\mathrm{n}=982)\end{array}$ & $\begin{array}{c}\text { SHIFU } \\
(n=113)\end{array}$ & $\mathbf{p}$ & SD & $\mathbf{n}$ & $\begin{array}{c}\text { NST } \\
(\mathrm{n}=177)\end{array}$ & $\begin{array}{l}\text { SHIFU } \\
(n=59)\end{array}$ & $\mathbf{p}$ & SD \\
\hline $\begin{array}{l}\text { Age at radiation }{ }^{1}, \text { mean } \pm \\
\mathrm{SD} \text {, median }(\mathrm{IQR})\end{array}$ & 1095 & $\begin{array}{c}70.3 \pm 6.5 \\
71.0(67.0 \\
75.0) \\
\end{array}$ & $\begin{array}{c}63.3 \pm 6.2 \\
63.6(59.3 \\
68.3) \\
\end{array}$ & $<0.001$ & 1.100 & 236 & $\begin{array}{c}67.2 \pm 6.1 \\
69.0(64.0 \\
71.0) \\
\end{array}$ & $\begin{array}{c}66.8 \pm 4.9 \\
67.6(63.7 \\
69.8) \\
\end{array}$ & 0.449 & 0.076 \\
\hline $\begin{array}{l}\text { Baseline PSA (pre- } \\
\text { radiation) }{ }^{1} \text { mean } \pm \mathrm{SD}, \\
\text { median (IQR) }\end{array}$ & 1077 & $\begin{array}{c}18.7 \pm 22.8 \\
11.9(7.3 \\
20.0) \\
\end{array}$ & $\begin{array}{c}12.9 \pm 20.0 \\
7.3(5.5 \\
12.2) \\
\end{array}$ & $<0.001$ & 0.270 & 236 & $\begin{array}{c}12.3 \pm 14.9 \\
9.4(6.2 \\
14.2) \\
\end{array}$ & $\begin{array}{c}10.4 \pm 7.1 \\
8.5(5.6 \\
13.9) \\
\end{array}$ & 0.362 & 0.167 \\
\hline $\begin{array}{l}\text { T stage (pre-radiation), }{ }^{1} \mathrm{n} \\
(\%)\end{array}$ & 1062 & & & $<0.001$ & & 236 & & & 0.626 & \\
\hline $\mathrm{T} 1$ & & $166(17.2)$ & $38(38.8)$ & $<0.001$ & 0.495 & & $47(26.6)$ & $18(30.5)$ & 0.419 & 0.088 \\
\hline $\mathrm{T} 2$ & & $482(50.0)$ & $52(53.1)$ & 0.564 & 0.061 & & $112(63.3)$ & $34(57.6)$ & 0.297 & 0.116 \\
\hline T3 & & $288(29.9)$ & $8(8.2)$ & $<0.001$ & 0.576 & & $18(10.2)$ & $7(11.9)$ & 0.602 & 0.054 \\
\hline $\mathrm{T} 4$ & & $28(2.9)$ & $0(0)$ & 0.101 & 0.245 & & $0(0)$ & $0(0)$ & - & - \\
\hline $\begin{array}{l}\text { Gleason score (pre- } \\
\text { radiation), }{ }^{1} \mathrm{n}(\%)\end{array}$ & 1063 & & & $<0.001$ & & 236 & & & 0.912 & \\
\hline$<6$ & 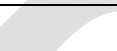 & $180(18.8)$ & $1(0.9)$ & $<0.001$ & 0.629 & & $1(0.6)$ & $1(1.7)$ & 0.317 & 0.107 \\
\hline 6 & & $212(22.2)$ & $46(43.0)$ & $<0.001$ & 0.455 & & $70(39.6)$ & $22(37.3)$ & 0.663 & 0.046 \\
\hline 7 & 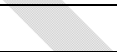 & $398(41.6)$ & $55(51.4)$ & 0.053 & 0.197 & & $100(56.5)$ & $33(55.9)$ & 0.916 & 0.011 \\
\hline $8-10$ & 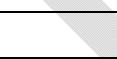 & $166(17.4)$ & $5(4.7)$ & $<0.001$ & 0.414 & & $6(3.4)$ & $3(5.1)$ & 0.405 & 0.084 \\
\hline GUROC risk, n (\%) & 1083 & & & $<0.001$ & - & 236 & & & 0.750 & - \\
\hline Low & & $122(12.4)$ & $31(30.7)$ & & & & $32(18.1)$ & $13(22.0)$ & & \\
\hline Intermediate & & $398(40.5)$ & $49(48.5)$ & & & & $114(64.4)$ & $35(59.3)$ & & \\
\hline High & & $462(47.1)$ & $21(20.8)$ & & & & $31(17.5)$ & $11(18.6)$ & & \\
\hline ADT pre-radiation, ${ }^{1} \mathrm{n}(\%)$ & 1095 & $397(40.4)$ & $23(20.4)$ & $<0.001$ & 0.447 & 236 & $52(29.4)$ & $15(25.4)$ & 0.370 & 0.089 \\
\hline
\end{tabular}


Nair et al

Role of salvage ablation post-radiation failure

\begin{tabular}{|l|c|c|c|c|c|c|c|c|c|}
\hline $\begin{array}{l}\text { Median followup (years), } \\
\text { median (95\% CI) }\end{array}$ & 1095 & $\begin{array}{c}11.6(11.3, \\
11.9)\end{array}$ & $\begin{array}{c}14.3(13.3, \\
16.3)\end{array}$ & $<\mathbf{0 . 0 0 1}$ & - & 236 & $\begin{array}{c}10.5(9.6, \\
11.1)\end{array}$ & $\begin{array}{c}13.5(11.5, \\
16.7)\end{array}$ & $<\mathbf{0 . 0 0 1}$ \\
\hline
\end{tabular}

${ }^{1}$ Included in propensity-score model; ${ }^{2}$ calculated using reverse Kaplan-Meier method; $\mathrm{p}<0.05$ and $\mathrm{SD}>0.10$ shown in BOLD. ADT: androgen deprivation therapy; CI: confidence interval; GUROC: Genitourinary Radiation Oncologists of Canada; IQR: interquartile range; NST: no salvage treatment; PSA: prostate-specific antigen; SD: standard deviation; sHIFU: salvage transrectal high intensity focused ultrasound. 